\title{
Morphological Group Theory of Material Structure
}

\author{
Ziqiang Zhou \\ Institute of Materials, Shanghai University, Shanghai, China \\ Email: zqzhou408@163.com
}

How to cite this paper: Zhou, Z.Q. (2018) Morphological Group Theory of Material Structure. Journal of Applied Mathematics and Physics, 6, 69-89.

https://doi.org/10.4236/jamp.2018.61008

Received: November 30, 2017

Accepted: January 2, 2018

Published: January 5, 2018

\begin{abstract}
The correct formulation and understanding of micro-images is one of the difficulties that occur to microstructures science today, which need to develop a new appropriate mathematics for micro-images of matter system. Here I study the image mathematics and physics description of micro-images of material system by topology, set theory, symbolic logic and show that there is a naturally morphological equation, that is a law of qualitative structure of matter system, the law of the unity of two kinds of morphological structure (Jordan and hidden structure), which can be used to describe not only the common feature of different correlated matter, but also to correct classify the micro-images into different classes, so that to study the morphology groups for materials science and Algebraic geometry. The morphology equation can be found a number of applications for the observation and analysis of micro-images of material system and other natural sciences, some important basic concepts of algebraic geometry can also be newly explained by the morphology equation, such as: 1) To construct the image-mathematical language and to construct the image mathematics model (IMM) for microstructures; 2) To construct complex geometric structures (Concave polygon) then analyze these complex shape structure by analytic geometry and algebraic geometry, to study complicated operators on complicated spaces; 3) A new explanation for the logical basis, concept definition and proof way of algebraic geometry and uses it to analyze morphological structure of the new and parent phase and the problem of Hodge's theory and structure type, and points out that there may be a counterexamples for Hodge's conjecture.
\end{abstract}

\section{Keywords}

Morphological Equation, Morphological Function, Algebraic Geometry Model, Complex Geometric Structures, Hodge Conjecture 


\section{Introduction}

Morphology is a branch in biology which dealing with the form and structure of organisms and their specific structural features. These concepts can also be used to describe the form and structure of the internal structure images of the different materials under microscope [1], which are of complex and have been changing. This kind of method provides a huge amount of valuable images for us to understand the internal structure of the material. But there exist still some difficult to be solved about how to explain and understand these images. One is how to classify these micro-images and to study the relation among the different shape kinds of complex micro-images? That is needed to find the unchanged laws in these changing micro-images [2]. The other is the relation between 2D images and $3 \mathrm{D}$ images. The region observed by microscopy is mostly a $2 \mathrm{D}$ micro area, the actual material is of $3 \mathrm{D}$ specimen, it is a basic problem of microstructure science to be solved how to reconstruct the 3D structure of specimen of material from 2D micro-images. In other words, how to construct an images mathematics model (IMM), which can to convert from 2D images to 3D images, from local region images to whole region images, from micro images to macro images, these are the basic problems to be solved for correlated matter science including materials science and biology. The development of basic research will enter an important leap from quantitative change to qualitative change [3].

Algebraic geometry developed a powerful way to study the shape of complex objects. This technique has become so useful that it can be generalized in many different ways, eventually leading to powerful tools that make great strides for mathematicians in classifying the various objects they encounter. Algebraic geometry has created an impressive edifice, but its logical basis is not solid, its concept is not well defined and the proof to be not sufficient, in some sense, there must be add some part with no any geometrical explanation [4].

As its name implies, algebraic geometry deals with both the concepts and methods of geometry and algebra. The different types of mathematicians has his emphasis to study the algebraic geometry problem, some focus on algebra, some focus on geometry, in which the advantage of algebraic methods can be far away from geometric intuition, then the study of $\mathrm{n}$-dimensional problem is not too difficult. But it also brings up some problems, it is easy to predict structure types, but it is difficult to construct these complex types. Therefore, it is of great scientific significance to develop a mathematical model that can carry out geometric and algebraic studies simultaneously.

The Hodge conjecture is a major unsolved problem in the field of algebraic geometry that relates the algebraic topology of a non-singular complex algebraic variety and the subvarieties of that variety. Predictably, the solution of Hodge's conjecture will find some basic internal relations among the three branches of mathematics, namely, analysis, topology, and algebraic geometry [5] [6] [7]. Using a nice phrase Atiyah said, "topologists used to study simple operators on complicated manifolds while analysts studied complicated operators on simple 
spaces." The time has arrived to study complicated operators on complicated spaces [8]. The algebraic geometry model required for morphological analysis is just such a kind of difficult question.

Here I want to discuss the construction about IMM for descriptions of the different complex structures for materials science and find some basic internal relations between the three branches of mathematics, namely, analysis, topology, and algebraic geometry. I begin with a morphological equation [9] [10] based Jordan curve theorem [11], then through introducing a method of set theory and symbolic logic [12] [13] to construct the model of algebraic geometry problem (AGM), the middle step in the process consists of finding a image-mathematical solution to the image problem by algebraic geometry and the final to interpret for these complex and changing images of different matters for morphology physics by the equation as an interdisciplinary image mathematics model (IMM).

\section{Morphological Equation and Morphological Group}

\subsection{Basic Concepts and Notation}

Concept is the first and the most important step in logic analysis; the intension and extension of the concept are determined by definition and classification respectively. The definition of image and the classification of the shape of the images are discussed.

\subsection{Lemma}

In mathematics the concept of image is defined by the mapping or function, a mapping (or function) $f$ from $S$ to $T$, where $S$ and $T$ are non-empty sets, is a rule which associates, with each element of $S$, a unique element of $T$. The set $S$ is the domain and $T$ is the co-domain of $f$. For $s$ in $S$, the unique element of $T$ that $f$ associates with $s$ is the image of s under $f$ and is denoted by $f(s)$.

The image of $s$ under $f$ is represented by the symbol $f(s)$,

$$
f: s \rightarrow f(s)
$$

Jordan curve theorem [11] calls a non-intersecting closed curve in a plane as simple closed curves $C$ and shows that any simple closed curve $C$ divides the points of the plane not on $\mathrm{C}$ into two distinct domains $\mathrm{A}$ and $\mathrm{B}$ (with no points in common) of which $C$ is the common boundary. The two sets of points are the set $\mathrm{A}$ of points inside $\mathrm{C}$ and the $\mathrm{B}$ outside $\mathrm{C}$. Points in the same set can always be joined by a curve lying entirely in the set. The theorem is a basic law in topology for describing manifolds.

\subsection{Definition}

Since the set $A$ of points inside a simple closed curve $C$ must be a connected region, I call the connected region as a morphological element (ME) [9], but the set $B$ of points outside the simple closed curve is also a connected region, so it is 
also a ME. To distinguish this two kinds of $\mathrm{ME}$, we can call the former connected region as Jordan ME (or $\llbracket J \rrbracket$ ), which can be used to describe the mor-

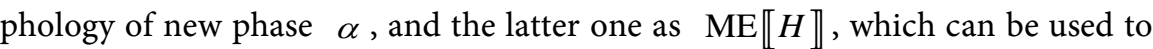
describe the morphology of the parent phase austenite $\gamma$ [10]. So the morphology $\mathrm{M}$ is consisted of two ME of $\llbracket H \rrbracket$ and $\llbracket J \rrbracket$,

$$
M=\llbracket H|z| J \rrbracket
$$

In the morphological Equation (2.2), « 》 denote a symbol of image operator, and $|z|$ stands for a morphological transformation defined by Jordan simple closed curve [10].

Such two kinds of ME may also be used to describe microstructure of other materials, which can be used as the image-mathematical description language $\mathcal{L} \mathbf{z}$, significantly improve the description of the micro-images.

Jordan curve theorem is a theorem of the form deduction system for image mathematics.

For discussing the problem of micro-images, a new image mathematics mode is used which combined about Jordan curve theorem [11], set theory [12], symbolic logic [13] (Venn diagrams) [14] p. 354 and ZhouYi [15] (The view of image number in Zhouyi-the ancient Chinese view of nature) and can be used as a common image description language $\mathcal{L} z$ for image mathematics model (IMM).

In order to discuss physical problem of matter-systems by morphological equations, we must also discuss if morphology equations is "true" for matter-systems, which involves the problems of notation, logic and proofs [12], morphology equation described how the formulas of the image of matter-system could be built up from component parts.

\subsection{Definition: Morphological Relation and Deduction Theorem for Image-Mathematics}

For a given observation region, $x \in M$, in which there is a simple closed curve $C$, it has the following properties

1) The morphological transformation $|z|$ (or function) defined by a simple closed curve $C$ (must divide the region $x \in M$ into two kind classes, the interior region $A$ and the exterior region $B$ );

$$
|z|=C
$$

2) The interior image Int. $M$ is defined by

$$
z \mid x \rrbracket=J \llbracket(x) \rrbracket
$$

it can also be called as morphological element-the Jordan ME $J \llbracket(x) \rrbracket$, sometimes abbreviated to $\llbracket J \rrbracket$ or $J$;

3) The exterior image Ext. $M$ is defined by

$$
\llbracket x \mid z=H \llbracket(x) \rrbracket ;
$$


it can also be called as ME $H \llbracket(x) \rrbracket$, sometimes abbreviated to $\llbracket H \rrbracket$ or $H$

4) of which the simple closed curve $C$ is the common boundary $|z|$.

$$
M=\llbracket H(x)|z| J(x) \rrbracket
$$

Given a micro-image we can know what objects are named by letter $|z|$, $J \llbracket(x) \rrbracket, H \llbracket(x) \rrbracket$ and can judge the truth or falsity of such "atomic" formulas.

Given any formulas $J \llbracket(x) \rrbracket$ and $H \llbracket(x) \rrbracket$ we can combine them in various ways to obtain new ones, the intended meaning of the new formulas can be captured by giving a truth table. One might judge if these formulas is true or false. The morphological equation can be used as a common image description language $\mathcal{L} z$ for image mathematics model (IMM).

The morphological transformation $|z|$ defined by simple closed curve $C$ can reflect simultaneously the characteristics of geometrical form and quantity relation.

Jordan closed-curve theorem is the basic theorem of the mathematical description language for image, which consists of several statements. Formula (2.3)-(2.6) are four sub-formula deduced from Jordan curve theorem, which can be called the deduction theorem of image-mathematics .

\subsection{Topology Structure for Morphological Group}

Example there are four curves in Figure 1, for any sets $A$ and $B$, there is a set whose members are exactly the pairs $\llbracket j, h \rrbracket$ with $j \in A$ and $h \in B$.

This is a group composed four curves with different geometry shape, but are all belong to the simple closed curve, which are the common feature of them,
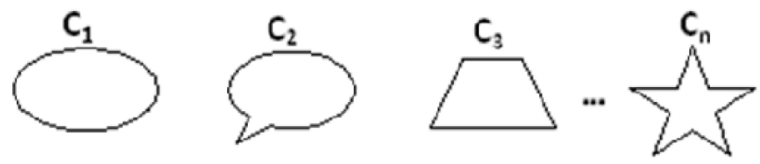

(a)
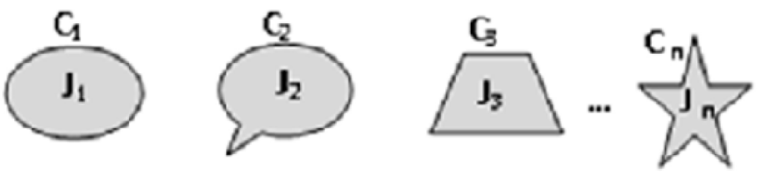

(b)

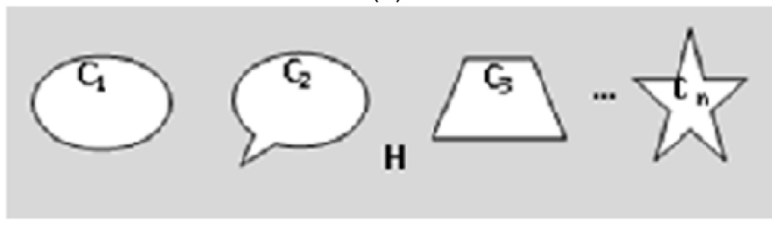

(c)

Figure 1. Two kinds of ME with different simple closed curve, which represented by the shaded areas. (a) the shape

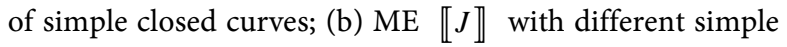
closed curves; (c) ME $\llbracket H \rrbracket$ with the same simple closed curves given by Jordan theorem and Venn diagram. 
i.e., invariance. Given one observation region, $x \in M$, in which there is a simple closed curve $C$, the morphological transformation $z$ (or function) defined by a simple closed curve $C$ must divide the region, $x \in M$ into two kind classes of the interior image-the Jordan $\mathrm{ME}, z \mid x \rrbracket=\llbracket J(x) \rrbracket$ and the exterior image $\llbracket x \mid z=\llbracket H(x) \rrbracket$, of which $C$ is the common boundary. $M(x)=\llbracket H(x)|z| J(x) \rrbracket$

Here ME 【J】 has the following the main properties: variability in geometry, invariability in topology and ordering in analytical geometry, which can be used to describe the complex and variable image of matter particle, and will play an important role in description for irregular shape of various complex micro-images.

\subsection{Definition Ordered pairs $\llbracket j, h \rrbracket$ is defined to be $\{\llbracket j \rrbracket, \llbracket j, h \rrbracket\}$}

We must prove that this definition succeed in capturing the desired unique decomposition property; The ordered pair $\llbracket j, h \rrbracket$ uniquely determine both what $j$ and $h$ are, and the order upon them.

Ordered pairs [12] p. 35.

we want to define a set $\llbracket j, h \rrbracket$ that uniquely encodes both what $\llbracket j \rrbracket$ and $\llbracket h \rrbracket$ are, and also what order they are in. In other words, if an ordered pair can be represented in two ways

$$
\sigma \in M: \llbracket j, h \rrbracket=\llbracket \alpha, \gamma \rrbracket \text { or } \llbracket j, h \rrbracket=\llbracket A, B \rrbracket
$$

then the representation are identical in the sense that $j=\alpha$ and $h=\gamma$ or $j=A$ and $h=B$ And in fact any way of defining $\llbracket j, h \rrbracket$ that satisfies this property (Jordan curve theorem) of unique decomposition will suffice. It will be instructive to consider first some examples of definitions lacking this property.

A morphological relation is a set of ordered pairs $\llbracket j, h \rrbracket$, which is defined by the morphological transformation $z$.

For such a relation $R$, we sometime write $j R_{z} h$ in place of $\llbracket j, h \rrbracket \in R_{z} \quad$ (2.8)

\subsection{Definition: Morphological Function}

Lemma Theorem about inverse function [12] p. 48.

Assume that $F: A \rightarrow B$, and that $A$ is nonempty

1) there exists a function $G: B \rightarrow A$ (a left inverse) such that $G \circ F=I_{A}$ is the identity function $I_{A}$ on $A$ iff $F$ is one-to-one.

2) there exists a function $H: B \rightarrow A$ (a right inverse) such that $F \circ H=I_{B}$ is the identity function $I_{B}$ on $B$ iff $F$ maps $A$ onto $B$.

Example Here I would like to describe the microstructures of micro-images, in Figure 1, Assume that $f: z \rightarrow x$, let $z$ be Jordan closed curve $C$ and $x$ a connected region $\mathrm{ME}$ of $M$, Apply the theorem about inverse function, the morphological transformation $Z$ will transform a simple closed curve to a connected region $J$ or $H$, that is

1) there exists a function $j: x \rightarrow Z$ (a left inverse) such that $j \circ f=I_{A}$ is 
the identity function $I_{A}$ on $A \llbracket j \rrbracket$ iff $F$ is one-to-one;

2) There exists a function $h: x \rightarrow z$ (a right inverse) such that $f \circ h=I_{B}$ is the identity function $I_{B}$ on $B$, iff $f$ maps $z$ onto $B \llbracket h \rrbracket$.

Why do we introduce the right inverse function $h: x \rightarrow z$ ? The reason is of when there exist more than one simple closed curve in the observation area $x$, (there exist four simple closed curves in Figure 1), we do not know that the connecting area $H$ corresponds to which simple closed curve $c=z$, so one must at first maps the connected region $H$ to one of the simple closed curves $z$, then to transform the simple closed curve $z$ into the connected region $H$. Therefore, the two inverse functions are different in nature, the former $j$ is one-to-one corresponding, belongs isomorphic corresponding; but the latter $h$ is a one-to-many correspondence, belongs to the homomorphism correspondence.

\subsection{Models [13] p. 100, Truth and Satisfaction}

We will use the following symbols: logical symbols (the connectives $\wedge, \vee, \cdots$; the quantifiers $\forall, \exists ; \cdots ;$ ) constant symbols $c$, function symbols $F$, relation symbols $R$.

Definition. A language $\mathcal{L}$ is a set consisting of all the logical symbols with perhaps some constant, function and/or relational symbols included. It is understood that the formulas of $\mathcal{L}$ are made up from this set in the manner prescribed above. Note that all the formulas of $\mathcal{L}$ are uniquely described by listing only the constant, function and relation symbols of $\mathcal{L}$.

We use $t\left(v_{0}, \cdots, v_{k}\right)$ to denote a term $t$ all of whose variables occur among $v_{0}, \cdots$, $v_{k}$

We use $\varphi\left(v_{0}, \cdots, v_{k}\right)$ to denote a formula $\phi$ all of whose free variables occur among $v_{0}, \cdots, v_{k}$

Definition. A model (or structure) $\mathfrak{A}$ for a language $\mathcal{L}$ [13] is an ordered pair $\langle A, \mathfrak{I}\rangle$, where $A$ is a nonempty set and $\mathfrak{I}$ is an interpretation function with domain the set of all constant, function and relation symbols of $\mathcal{L}$ such that:

1) if $c$ is a constant symbol (a given simple closed curve), then $\Im(c) ; \Im(c)$ is called a constant i.e., a given $\llbracket J \rrbracket$

2) if $F$ is an $m$-placed function symbol, then $\Im(F)$ is an $m$-placed function on $A$

3) if $R$ is an $n$-placed relation symbol, then $\Im(F)$ is an $n$-placed relation on A.

A is called the universe of the model $\mathfrak{A}$. We generally denote models with Gothic letters and their universes with the corresponding Latin letters in boldface. One set may be involved as a universe with many different interpretation functions of the language $\mathcal{L}$. The model is both the ME and the interpretation function.

The importance of Model Theory lies in the observation that mathematical objects can be cast as models for a language. 


\subsection{Natural Models [12] for Morphology}

The class and structure of $\llbracket J \rrbracket$ morphology.

I want to consider the question of whether there can be a set $M$ that is in a certain sense a miniature model of the class $V$ of all sets. Consider a formula $\sigma$ of the language of set theory; for example $\sigma$ might be one of our axioms. We can convert $\sigma$ to a new formula $\sigma^{M}$ by replacing expressions $\forall x$ and $\exists x$ by $\forall x \in M$ and $\exists x \in M$. Then the proposition (true or false) that $\sigma$ asserts of $\mathrm{V}$ is asserted by $\sigma^{M}$ of just $M$.

Example According to the morphological equation, in a observation region where a simple closed curve exists, there is an ordered pair $\sigma \in M: \llbracket j, h \rrbracket$ $\llbracket j, h \rrbracket$, which is introduced by the simple closed curve theorem.

Let $\sigma$ be the pairting axiom,

$$
\forall j \forall h \exists z \forall x[x \in z \Leftrightarrow x=j \text { or } x=h] .
$$

Then $\sigma^{M}$ is

$$
(\forall j \in M)(\forall h \in M)(\exists z \in M)(\forall x \in M)[x \in z \Leftrightarrow x=j \text { or } x=h] .
$$

This says that you can take any $j$ and $h$ inside the set $M$, and be assured of the existence of a set $z$ inside the set $M$ whose members belonging to the set $M$ are exactly $j$ and $h$.

\section{Algebraic Geometry Model (AGM)}

Algebraic geometry developed a powerful way to study the shape of complex objects. This technique has become so useful that it can be generalized in many different ways, eventually leading to powerful tools that make great strides for mathematicians in classifying the various objects they encounter. Algebraic geometry has created an impressive edifice, but its logical basis is not solid, its concept is not well defined and the proof to be not sufficient. In some sense, there must be added some part with no any geometrical explanation.

In order to solve these problems we need to introduce the methods in mathematical models, these problems must first be abstracted into a mathematical problem, then solved by the pure mathematical method, and finally explained the original question through the explanation. A usable mathematical model, it must be able to describe at least the structural form and quantity relationship required by the original algebraic geometry, and also be helpful to solve the key problems that have not been solved previously. In order to describe the classification problem of complex geometry structure by morphological equation or morphological group theory, there are three questions have to be answer: i.e., How to construct a complex shape structure; How to analyze these complex shape structure; How to analyze complex shape structure with algebraic geometric methods. Ellipse and polygon are two common basic geometrical shapes, so how to transform them into complex geometry structure is the first problem we need to solve. 


\subsection{How to Construct a Complex Shape Structure (from Convex Set to Non-Convex Set)}

In order to define the morphological transformation group, we consider the morphological equation as the basic equation, Jordan curve as the morphological boundary transformation $z$, let it generate the singular boundary group, then define the homomorphism of the boundary, to obtain the singular morphological cycle group and the morphological group.

Example 2. To transform ellipse into complex geometry structure.

In the mathematical field of algebraic topology, the fundamental group is a mathematical group associated to any given pointed topological space that provides a way to determine when two paths, starting and ending at a fixed base point, can be continuously deformed into each other. It records information about the basic shape of the topological space. The fundamental group is the first and simplest homotopy group.

The fundamental group is a topological invariant: homeomorphic topological spaces have the same fundamental group. We can use the homotopy group approach [11] to construct complex shape structures.

\subsection{Transformation Rule}

One can use of homotopy group methods to construct complex geometric structures (Concave polygon) from elliptical shape of $\llbracket J \rrbracket$ ME.

The transformation rule is as follows: the following transformation $|z|$ of the boundary is called the allowed transformation of the boundary:

1). when $a^{r-1}=a^{r}, a^{r-1} a^{r} \cdots$ to become $\cdots a^{r} \cdots$ or vice versa.

2). When $a^{r-1}, a^{r}, a^{r+1}$ unfolds into the simple closed curve, the boundary $\cdots a^{r-1}$, $a^{r}, a^{r+1} \cdots$ to become $\cdots a^{r-1}, a^{r+1} \cdots$ or vice versa.

Specifically, the transform operation is as follows:

1) replace the middle third of one side of ellipse of $\llbracket J \rrbracket \mathrm{ME}$, one side into four edges, this forms a shape as shown in the second diagram, which $n_{\max }$ is 4 . Fixed the two points $a_{0}, a_{2}$ above the ellipse arc, makes the ellipse arc deformation into $a_{0}, a_{1}, a_{2}$ Convex polygon and non-convex set.

2) repeat this construction to obtain the figure shown in the third diagram, $n_{\max }$ is 6 . and the diagram $4, n_{\max }=8>6$, The shape of the mountain.

The graphs produced by these rules are shown in Figure 2 .

The Koch curve is an example about polygon to be transformed into complex geom. The structural changes can be referred to in the literature for details [14].

\subsection{Definition: Morphological (Transformation) Group for $\llbracket J \rrbracket M E$}

A group $G$ is a set, which is a simple closed curves set together with two operations (or more simply, functions), one called morphological transformation $z$. $G \times$ $G \rightarrow G$ and the other called the inverse $i: G \rightarrow G$. These operations obey the following rules: 


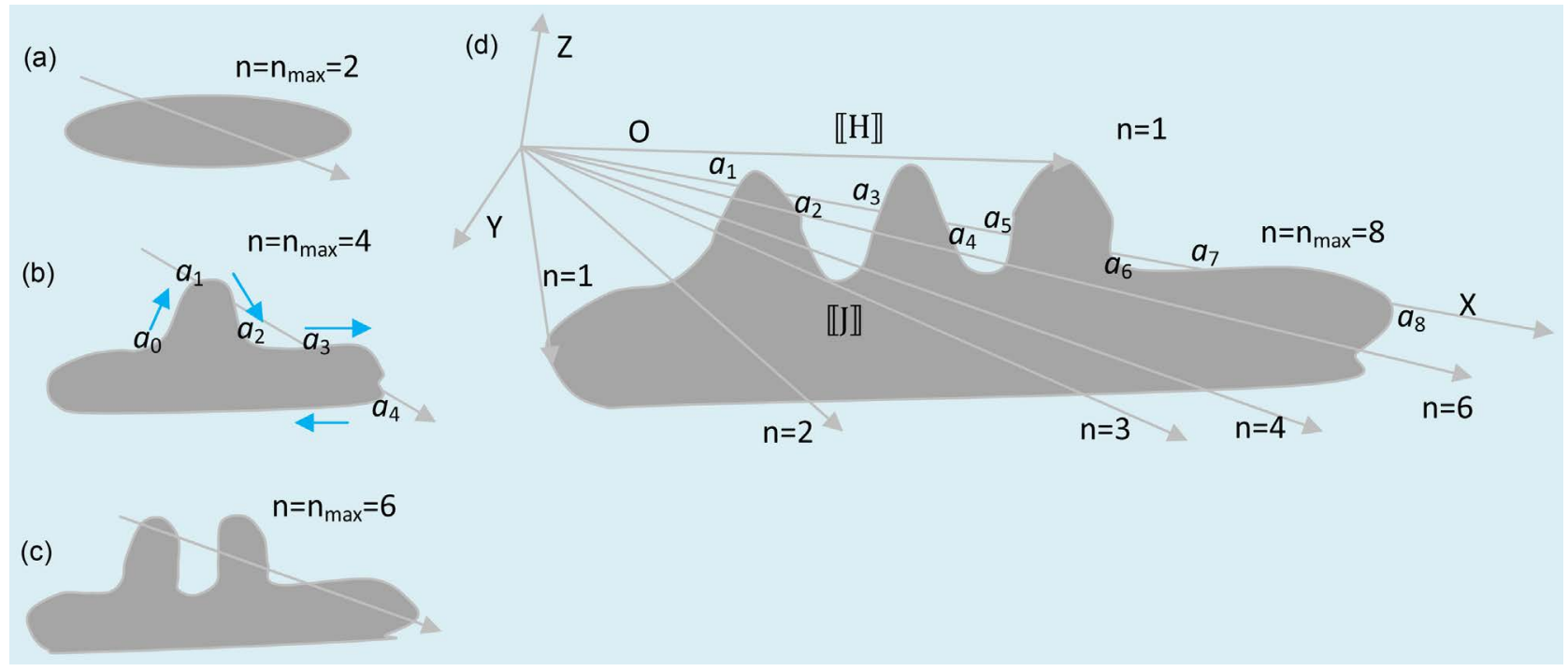

Figure 2. The relations between different shapes of $\llbracket J \rrbracket$ ME image; (The shape of the mountain or coastline).

1) Associativity: For every $g, h$ and $k \in G_{z},(g h) k=g(h k)$.

2) Identity: There is an element e in the group $G_{J}$ such that for every $g \in G_{z}$, $g e=g=e g$

3) Inverse: For every $k \in G_{z}, g g^{-1}=e g^{-1} g$

Proof: interpretation according Venn diagram [14].

1) If there exist three simple closed curves $c_{v}$ in the Venn diagram, $g, h$ and $k$ in the one observation region, $x \in \llbracket J \rrbracket \in M$, for every $g, h$ and $k \in \llbracket J \rrbracket \in M$, the associativity $(g h) k=g(h k)$ can be interpreted by $(g \cup h) \cup k=g \cup(h \cup k)$

2) and 3) can be interpreted by Theorem about inverse function.

Definition: The above operation is a multiplication operation, and the collection of elements of multiplication group belongs to the power set for $\llbracket J \rrbracket \mathrm{ME}$, which can be expressed as following rule:

The power of multiplicative group elements: $a^{n}=a \cdots a ; a^{0}=e, a^{-n}=(-a)^{n}$;

The exponential rule is $a^{n}=a \cdots a$

\subsection{How to Analyze These Complex Shape Structure: Analytic Geometry of Space}

Analytic geometry analysis of object structure is one of the important problems in algebraic geometry. There are two aspects, one is the application of the intersection method, the other is how to analyze the data generated by the intersection method-order structure theory problem. The former transforms the object structure graph into a set of numbers, while the latter is to recognize these numbers by order structure theory.

The principle of analytic geometry Based upon the principle of analytic geometry of space, the structure of a simple closed curve (or spatial surface) of a $\llbracket J \rrbracket$ (i.e., a fixed field $\mathrm{k}$ ) can be studied by the intersection number $\boldsymbol{n}$ of a ray with respect to the curve of the $\llbracket J \rrbracket$, such an operation can transform a 
simple closed curve into a set of numbers, which can be applied to describe the structure of the curve as feature parameters of the curve or $\llbracket J \rrbracket$. [9] [10]

Example There exist four shapes of different geometries In Figure 2, The intersection method converts each closed curve into a set of numbers, For the Figure 2(d), the intersection point of a ray with the simple closed curve can be represented by different symbols $a_{1}, a_{2}, \cdots, a_{k}$, ordering from small to large, which are $1,2,3, \cdots, 8$, and its maximum value is $n_{\max }$ which can be regarded as an embedded relation

$$
E: k \rightarrow t\left(a_{1}, a_{2}, \cdots, a_{k}\right) \text { for } \llbracket J \rrbracket,
$$

so the minimum number of intersection points is one, the maximum intersection number is $n_{\max }$ which represents a K-place relation $R \subset^{k} \llbracket J \rrbracket$. Let $R$ be a k-place relation on $J$, and tern $t\left\langle a_{1}, a_{2}, \cdots, a_{k}\right\rangle \in^{k} \llbracket J \rrbracket$.

We can get four different number of intersection points for four figure, the maximum number of intersections $n_{\max }$ are 2, 4, 6, 8 respectively, which reflects the characteristics of their geometrical shape; the larger the $n_{\max }$ the more complex the geometry of the graph is. The $n_{\max }$ can be described a partial odering: $2<4<6<8$ In Figure 2.

This relationship is a new relation produced by the analysis of the intersection method to morphological elements $\llbracket J \rrbracket$, Our first application of intersection points methods will be to the construction of the ordinal numbers, order structure and order type [12]

If $<$ is some sort of ordering on $A$ (at least a partial ordering) and $t \in A$, then the set

$$
\operatorname{seg} t=\{x \mid x<t\}
$$

called the initial segment up to $t$.

$$
\text { An embedding function } E: \mathbb{Z} \rightarrow \mathbb{Q} \text { defined by } E(a)=[\langle a, 1\rangle]
$$

For example, natural numbers $\omega$ was ordered by $\in$, and consequently we had for $n \in \omega$, seg $n=\{x \mid x \in n\}=n$. So we can define an ordered structure $\langle A,<\rangle$.

The ordinal number of $\langle A,<\rangle$ is its $\in$-image. An ordinal number is a set that is the ordinal number of some well-ordered structure.

Assume that $<$ is a well ordering on $A$ and take for $\gamma(x, y)$ the formula: $y=$ $\operatorname{ran} x$. The transfinite recursion theorem then presents us with a unique function $E$ with domain $A$ such that for any $t \in A$ :

$$
E(t)=\operatorname{ran}(E\lceil\operatorname{seg} t)=E \llbracket \operatorname{seg} t \rrbracket=\{E(x) \mid x<t\}
$$

Let $\alpha=\operatorname{ran} E$; we will call $\alpha$ the $\in$-image of the well-ordered structure $\langle A,<\rangle$.

Definition: A partial ordering is a relation $R$ by the ordering from small to large, meeting the following two conditions: [12]

1) $R$ is a transitive relation: $x R y \& y R z \Rightarrow x R z$.

2) $R$ is irreflexive : It is never the case that $x R x$. 
Example To get some idea of what the $\in$-image might look like, take the three-element image set $A=\{r, s, t\}$,

where $r=4<s=6<t=8$. then

We calculate in Figure $2 E(r)=\{E(x) \mid x<r\}=4$; for Figure 2(b)

$E(s)=\{E(x) \mid x<s\}=6$; for Figure 2(c)

$E(t)=\{E(x) \mid x<t\}=8$. for Figure 2(d)

Definition A well ordering on $A$ is a linear ordering on $A$ with the further property that every nonempty subset of $A$ has a least element. Well orderings are significant because they can be used to index constructions that proceed "from the bottom up", where at every stage in the construction (except the last) there is a unique next step. This idea is made precise in the transfinite recursion theorem, which we will encounter presently.

Limit ordinal $\lambda$-limit of sequence the intersection number $n$ of a ray with the simple closed curve can be applied to describe the structure of the curve as feature parameters of the curve, the $n_{\max }$ depends the structure of the $\llbracket J \rrbracket \mathrm{ME}$, we call it as limit ordinal $\lambda$.

Example: A partial ordering is a special sort of relation, Let $M$ be any fixed set, and let $\subset_{\mathrm{M}}$ be the relation of strict inclusion on subsets of $M$ : The partial ordering of the morphological set $M$ is as follows, based on spatial inference relations (Figure 2)

$$
\left.\subset_{\mathrm{M}}=\{<J, H\rangle \mid J \subseteq H \subseteq M \text { and } J \neq H\right\}
$$

(More generally, we could seek to define an "order type" ot $\langle A, R\rangle$ whenever $R$ was a partial ordering on $A$. The order type should measure the "shape" of $R$, in the sense that

$$
\text { ot }\langle A, R\rangle=\text { ot }\langle B, S\rangle \text { iff }\langle A, R\rangle \cong \text { ot }\langle B, S\rangle
$$

This can be done.

These concepts will play an important role in the study of algebraic geometry.

\section{Algebraic Geometry}

The method mentioned above is the analysis of the shape structure from analytic geometry, which provides us with a lot of shape information, but it has not yet been linked to the algebraic structure of the graph. To solve this problem, we must introduce the basic algebraic equation, into algebraic geometry.

\subsection{The Relation between an Algebraic Cycle and Jordan Closed Curve}

Algebraic geometry is a branch of mathematics, classically studying zeros of multivariate polynomials. Modern algebraic geometry is based on the use of abstract algebraic techniques, mainly from commutative algebra, for solving geometrical problems about these sets of zeros. Hodge theory is a central part of algebraic geometry, Hodge class is deal with the classes $c l(Z)$ of algebraic cycles. By Chow's theorem, on a complex projective variety, algebraic cycles are the 
same as closed analytic subspace [16].

In order to make the morphological equation as a mathematical model of algebraic geometry, one condition has to be seek the intrinsic relationship between the morphological equation and the algebraic geometry, and to give a reasonable explanation to the algebraic geometry problem by the morphological equation, including the structure element, morphological relation and morphological function.

Cycle (In graph theory) [14] in a graph, a cycle is a sequence $v_{0}, e_{1}, \cdots, e_{k}, v_{k}$ $(k \geq 1)$ of alternately vertices and edges (where $e_{i}$ is an edge joining $e_{i-1}$ and $e_{i}$ ), with all the edges different and all the vertices different, except that $v_{0}=v_{k}$.

Jordan curve is a simple closed curve, which is continuous plane curve that has no ends (or in other words, that begins and ends at the same point) and not intersect itself.

These theory both describe the structure of the simple closed curve, but there exist the important difference, the former emphasizes the algebraic structure, which is connected with the algebraic geometry; but the latter emphasizes the topological structure, directly connected with the Jordan closed curve theorem, which is a basic theorem of topology, and is more advantageous to the topological structure of the analytic form, the theorem is a basic law in topology for describing manifolds.

\subsection{Definition: Arithmetic of Order Types-Morphological Field}

Definition an order type is the isomorphism type of some linearly ordered structure.

We will use Greek letter $\rho, \sigma, \cdots$ for order types. Any member of an order type $\rho$ is said to be a linearly ordered structure of type $\rho$. First we want to define addition of order types, the basic idea is that $\rho+\sigma$ should be the order type "first $\rho$ and then $\sigma$." For the real definition of $\rho+\sigma$, first select $\langle A, R\rangle$ of type $\rho$ and then $\langle B, S\rangle$ of type $\sigma$ with $A \cap B=\varnothing$, then define the relation $R \oplus S$ by

$$
R \oplus S=R \bigcup S \bigcup(A \times B)
$$

Finally we define $\rho+\sigma$ by $\rho+\sigma=\operatorname{it}\langle A \cup B, R \oplus S\rangle$

The idea is that we want to order $A \cup B$ in such a way that any member of $A$ is less than any member of $B$. but within $A$ and $B$, we order according to $R$ and $S$. [12]

Example: $A$ straight line intersects with a simple closed curve, which is the common boundary between $X \sim \llbracket J \rrbracket$ and $\mathbb{C} \sim \llbracket H \rrbracket$, and links the structure of the $\llbracket J \rrbracket$ and $\llbracket H \rrbracket$ to the straight line: $f: X \rightarrow S$. The numbers of the intersection points is $n$, Some of the segments in $J \llbracket J \rrbracket$, some of the line in $\llbracket H \rrbracket$, respectively, using two different symbols $p, q$, the total line is equal to the sum of the two segments.

$$
p+q=n
$$


Therefore the group operations of the Figure 3 can also be expressed as an addition groups. Among them

$$
\begin{gathered}
p=p_{1}+\cdots+p_{4} \in X \sim \llbracket J \rrbracket \text { and } q=q_{1}+\cdots+q_{4} \in \mathbb{C} \sim \llbracket H \rrbracket, \\
\sum: p_{1}+\cdots+p_{4}+q_{1}+\cdots+q_{4}=n
\end{gathered}
$$

These symbols $p$ and $q$ are belong to two different morphological structure elements $\llbracket J \rrbracket$ and $\llbracket H \rrbracket$ in morphological equation. This representation method also has a corresponding relationship, but the line segment can be multiple, so the corresponding relationship is not a one-to-one isomorphism corresponding relationship, but belong to the homomorphism.

It has been shown that the structure of the graph can be represented by a multiplicative group, and now it can also be represented by an additive group, the boundary is belong to a set of boundaries with multiplication and addition, belonging to a morphological ring, or as a morphological field. It has been shown that the existence of splitting decomposition, $p+q=n$.

\subsection{Concept and Simplest Properties of Algebraic Functions}

The relationship between algebraic field and morphological field [4].

Algebraic fields in algebraic geometry: Let $\mathbb{K}$ be an arbitrary commutative field, say, the field of complex numbers. The elements of $\mathbb{K}$ are called constants. Let $u_{1}, \ldots, u_{n}$ be indeterminates, or, more generally, arbitrary quantities in an extension field of $\mathrm{K}$, between which there are no algebraic relations with constant coefficients. The field of rational functions of $u_{1}, \ldots, u_{n}$ is denoted by $\mathbb{K}(u)$ or $\mathbb{P}$. We denote any element of an extension field of $\mathbb{K}(u)$ by $\omega$ and regard it as an algebraic function of $u_{1}, \ldots, u_{n}$ when it satisfies an algebraic equation $f(\omega)=0$ with (not identically vanishing) coefficients in $\mathbb{K}(u)$. Among the polynomials $f(z)$ with the property $f(\omega)=0$ there is a polynomial of least degree $\varphi(z)$, and one can prove algebraically that it has the following properties: 1$)$ $\varphi(z)$ is uniquely determined up to a factor in $\mathbb{K}(u) .2) \varphi(z)$ is irreducible. 3)

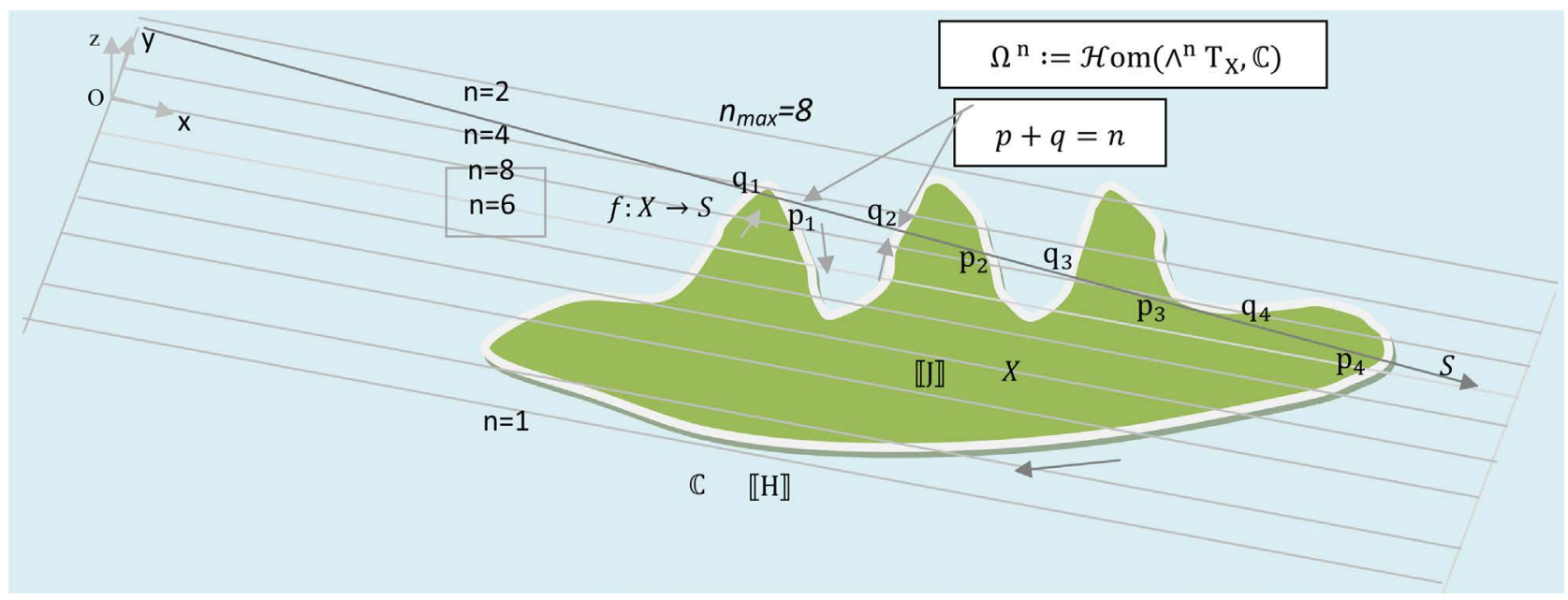

Figure 3. The algebraic geometric model for the existence of an integer Hodge class. 
Any polynomial $f(z)$ in $\mathbb{P}[z]$ with the property $f(\omega)=0$ is divisible by $\varphi(z)$. 4) For a given non-constant irreducible polynomial $\varphi(z)$ there is an extension field $\mathbb{P}(\omega)$ in which $\varphi(z)$ possesses a zero $\omega .5)$ The field $\mathbb{P}(\omega)$ is uniquely determined by $\varphi(z)$ up to isomorphism, i.e., if $\omega_{1}$ and $\omega_{2}$ are two zeroes of the same polynomial $\varphi(z)$, which is irreducible over $\mathbb{P}(\omega)$, then one will have $\mathbb{P}\left(\omega_{1}\right) \cong \mathbb{P}\left(\omega_{2}\right)$, and this isomorphism will leave all elements of $\mathbb{P}$ fixed and take $\omega_{1}$ to $\omega_{2}$. Polynomial Mapping $F, \mathrm{C}^{\mathrm{n}} \rightarrow \mathrm{C}^{\mathrm{n}}$

If $F$ is a field, $f(x)$ is a polynomial with a number greater than 0 in $F[x]$, then there is an extension of $F$, which causes $f(x)$ to have a root in the expansion field.

Now we can use the morphological equation to explain the algebraic field.

There are following properties about images of $\llbracket J \rrbracket \mathrm{ME}$, which are belongs to Jordan simple closed curves in topology, the relation $R$ between two images is of invariance in topology. In order to distinguish the geometry characteristics of image structures, we can use analytic geometry method through the maximum intersect point number $n_{\max }$, and use the algebra factor theorem to analyze images of $\llbracket J \rrbracket M E$, and classify the images into different classes [9] [10], According to the algebra factor theorem the mathematical equation describing the simple closed curves $\llbracket J \rrbracket$ ME must satisfy the following conditions:

As algebraic geometry deals with both geometric and algebraic concepts and methods, the following conditions must be satisfied to use polynomial equations to express the structure of the geometry:

1) According to analytic geometry it must satisfy the maximum intersection point, that is, the limit ordinal number $\lambda$ is not greater than the maximum number of $n_{\max }: \lambda \leq n_{\max }$

2) According to Fundamental theorem of algebra, the equation $f(z)=0$ cannot have more than $\mathbf{n}$ distinct roots, to use polynomial equations to express the structure of the geometry, the degree of $n_{k}$-order polynomials must not be less than $n_{\max }: n_{\max } \leq n_{k}$

To meet simultaneously both of these conditions of geometric and algebraic concepts, the degree of $n$-order polynomial must be equal to $n_{\max }$.

In order to satisfy the conditions of both geometry and algebra, the geometrical order structure should correspond to the order structure of algebra, i.e., there exists an equivalence relation: $\lambda \leq n_{\max } ; n_{\max } \leq n_{k}$; so the degree of $n$-order polynomial must be equal to $n_{\max }$.

Proof: Condition (1) has been proved in analytic geometry analysis (see Figure 2(d));

Condition (2) need to apply Fundamental theorem of algebra.

Jordan curve theorem is a basic law in topology for describing manifolds. There is some correspondence relation between algebraic geometry and morphological equation, and between algebraic cycle and Jordan simple closed curve, and between the algebraic manifold K-field and topological structure $\llbracket j \rrbracket$. This way of thinking will integrate the topology, analytic geometry and al- 
gebraic geometry, which will help to discover the intrinsic relationship among the three branches.

Fundamental theorem of algebra [14] p. 128 states that for every polynomial equation.

$$
a_{n} z^{n}+a_{n-1} z^{n-1}+\cdots+a_{1} z+a_{0}=0,
$$

where the $a_{i}$ are real or complex numbers and $a_{n} \neq 0$, has a root in the set of complex numbers.

It follows that,

if $f(z)=a_{n} z^{n}+a_{n-1} z^{n-1}+\cdots+a_{1} z+a_{0}$, there exist complex numbers $a_{1}, a_{2}, \cdots, a_{n}$ such that

$$
f(z)=a_{n}\left(z-a_{1}\right)\left(z-a_{2}\right) \cdots\left(z-a_{n}\right) .
$$

Hence the equation $f(z)=0$ cannot have more than $n$ distinct roots.

For a given coordinate $\mathrm{O}-\mathrm{x}$ y in Figure 2(d) and Figure 3, where $a_{1}, a_{2}, \cdots, a_{n}$ represent the coordinate $x$ of different intersecting points of a ray with the Jordan simple closed curve C, i.e., which is the common boundary of $\llbracket j \rrbracket$ and $\llbracket h \rrbracket$.

When the ray coincide with the coordinate $x$, the y coordinate of different intersecting points of a ray with the Jordan curve $\mathrm{C}$ is equal to 0 , the basic object of algebraic geometry is a set of common 0 points of a family of polynomial equations. i.e.,

$$
y=f(x)=\left(x-a_{1}\right)\left(x-a_{2}\right) \cdots\left(x-a_{n}\right)=0
$$

To meet both of these conditions, the degree of order of the polynomial must be equal to $n_{\max }$, the order of the above equation must be greater than or equal to $n_{\max }$ in Figure 3.

When the rays are not coincide with the coordinate $x$, the $y$ coordinate of different intersecting points of a ray with the Jordan curve $C$ is not necessarily equal to 0 , the algebraic function $y=f(x)$ of $u_{1}, \ldots, u_{n}$ i.e.,

$y=f(x)=\left(x-u_{1}\right)\left(x-u_{2}\right) \cdots\left(x-u_{n}\right)$ is not necessarily equal to 0, but the for the $\mathrm{O}$-xyz coordinate system, the third coordinate $z$ on the plane XY is always equal to 0 , the function equation of Jordan curve $C$ can be expressed as $z=0$, so for a given coordinate $\mathrm{O}-\mathrm{x} \mathrm{z}$ in Figure 2(d)

$$
z=g(x)=\left(x-a_{1}\right)\left(x-a_{2}\right) \cdots\left(x-a_{n}\right)=0
$$

It reflects the relationship of the different slice categories of the same manifold.

Therefore we can let the functions $f: X \rightarrow Y$, and $g: Y \rightarrow Z$ can be composed to yield a function $F$ which maps $X$ in $X$ to $g(f(x))$ in $Z$. Intuitively, if $z$ is a function of $y$, and $y$ is a function of $x$, then $z$ is a function of $x$. The resulting composite function is denoted $g \circ f: X \rightarrow Z$, defined by $(g \circ f)(x)=g(f(x))$.

$$
z=(g \circ f)(x)=g(f(x))
$$

For the coordinate system selected in Figures 2(d) and Figure 3, the other 
two coordinates $Y$ and $Z$ value of $a_{1}, a_{2}, \cdots, a_{n} \in \llbracket j \rrbracket$ on $X$ coordinate should be 0 .therefore the basic object of algebraic geometry is a set of common 0 points of a family of polynomial equations, i.e.,

$$
\varphi(z): z=F(x, y)=A f(x)+B g(x)=0
$$

i.e., Zeros of simultaneous polynomials. The results from the above expression can explain the main properties of Noether's fundamental theorem and slice theory.

\subsection{Replacement Axioms and the Function Classes [12] p. 179}

Morphological equation is also a law of qualitative structure of matter systems, which characterize the essential nature of the morphological image of matter structures. These characterizations are in variably qualitative in nature, for the

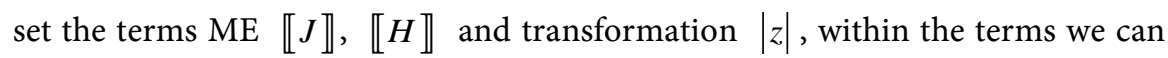
be developed more detailed morphological image knowledge by introducing analysis method of geometry and algebra. The basic object of algebraic geometry is a set of common 0 points of a family of polynomial equations. Their essence can often be captured in very short, very general statements. So that we can use the set of natural number to analysis the structure of morphological element set.

In other words there is a law of morphology in things or micro-images, that is, the law of the unity of $\llbracket H \rrbracket$ and $\llbracket J \rrbracket M E$ which is the fundamental law of nature morphology and a law of qualitative structure of matter science and therefore also the fundamental law of thinking in images.

The terms described by morphological equation are all composed of two kinds of $\mathrm{ME} \llbracket H \rrbracket$ and $\llbracket J \rrbracket$ and morphological transformation $Z$, on which a whole matter science operates. One might judge these general laws, due to their limited specificity, as making relatively little contribution to the sum of a science, were it not for the historical evidence that shows them to be results of the greatest importance.

Therefore the algebraic geometry model based on morphological equation can used to explain the structure of Hodge classes.

\section{Morphological Model and Hodge Conjecture}

\subsection{The Existence of Hodge Decomposition $\Omega^{n}=\oplus \Omega^{p, q}$}

According the morphology equation the structure in Figure 3 includes two parts $M=\{<\mathbb{C}, X>\mid \mathbb{C} \sim H, X \sim J\}$. for the coordinates selected, the numbers of the intersection point $a_{1}, a_{2}, \cdots, a_{n}$ of a ray with the manifold $X$ or K-fields is a certain integer $(z)$ that depends not only on the structure of the shape of $\llbracket J \rrbracket$, but also on the position and direction of the ray. For a given figure the maximum numbers of the intersection point $n_{\max }$ is only one, for Figure 3. the $n_{\max }$ is 8 .

The numbers of the intersection point $a_{1}, a_{2}, \cdots, a_{n}$ of a ray with the manifold $X$ or K-fields can be divided two classes, of which a class $p$ composed the directed line-segments of $1-2,3-4,5-6,7-8$ is in $\llbracket J \rrbracket$, the other $q$ composed of the 
directed line-segments $0-1,2-3,4-5,6-7$ is in form $\llbracket H \rrbracket$ or $\mathbb{C}$. There exist relation $p+q=n$.

$$
M=\{\langle q, p>| q \in h, p \in j \text { and } p+q=n\}
$$

which means there's an additive group here. The symbolic representation of the outer direct product of an additive group is as $Z_{2} \oplus Z_{2} \cong G$. This provides an explanations for the existence of Hodge's decomposition.

Such a morphological structure induces an action of the group $\llbracket H \rrbracket$ or $\mathbb{C}^{*}$ on $\llbracket J \rrbracket$ or the tangent bundle $T_{x}$, with $\lambda \in \mathbb{C}^{*}$ acting by multiplication by $\lambda$. The $X$ is an orientable manifold, the orientation of the manifold is expressed with arrowhead in Figure 2(b) and Figure 3. The arrowhead stipulated a clockwise direction.

\subsection{Kähler Manifold}

In mathematics and especially differential geometry, a Kähler manifold is a ma-

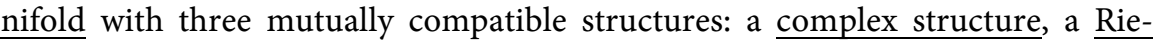
$\underline{\text { mannian structure, }}$ and a symplectic structure. Hodge theory is a central part of algebraic geometry, proved using Kähler metrics.

Morphological models are all composed of two kinds of ME $\llbracket H \rrbracket$ and $\llbracket J \rrbracket$ and morphological transformation $|z|$, it can describe manifold with two mutually compatible structures: a complex structure and a symplectic structure, but it is not necessarily to satisfy the continuity requirements of differential structures, because Jordan simple closed curves are not necessarily continuous and differentiable everywhere, there may be exist singularities in the curve.

\subsection{Hodge Type $(p, p)[7]$}

[7] From Figure 3 we can see that The numbers of the intersection point $a_{1}, a_{2}, \cdots, a_{n}$ of a ray with the manifold $X$ or K-fields can be divided two classes $<q, p>$, There are natural numbers $\mathbb{Z}$, and there exist relation $p+q=n$, and $p=$ $q$, so $<q, p>\sim<p, p>$.

\subsection{On the Locus of Hodge Classes Hodge Filtration [7]}

Let $S$ be a complex algebraic variety and $\left\{X_{s}\right\}_{s \in S}$ a family of nonsingular projective varieties parametrized by $S$ : the $X_{s}$ are the fibers of $f: X \rightarrow S$, with $X$ projective and smooth over $S$. Fix $s \in S$, an integer $p$, and a class $h \in H^{2 P}\left(X_{s}, \mathbb{Z}\right)$ of Hodge type $(p, p)$.

Let $U$ be an open, simply connected neighborhood of $s$. The $H^{2 P}\left(X_{s}, \mathbb{Z}\right)$, $t \in S$, form a local system on $S$, necessarily trivial on $U$, so that for $t \in U$ they can all be identified with $H^{2 P}\left(X_{s}, \mathbb{Z}\right)$. The Hodge filtration $\mathcal{F}_{t}$ of $H^{2 P}\left(X_{t}, C\right), t \in U$, can be viewed as a variable filtration on the fixed complex vector space $H^{2 P}\left(X_{s}, C\right)$. It varies holomorphically with $t$. It follows that the locus $T \in U$ where $h$ remains of type $(p, p)$, i.e., in $\mathcal{F}^{p}$, is a complex analytic subspace of $U$. 
From Figure 3 we can see that such an open, simply connected neighborhood $U$ of $S$ is really exist there.

But if $n$ is odd, this would imply for instance that $p$ plus $q$ equals odd, this means that $q$ and $p$ are not always the same even number or always the same as odd number, so $\langle q, p>$ and $\langle p, p>$ cannot be equivalent. The Hodge filtration has to be slightly corrected [17].

\subsection{There May be a Counterexample [16] [17]}

The above analysis involves the ordinal structure, the concept of partial order includes transitivity and irreflexive (2.8). In order to prove the existence of irreflexive, the paper uses the least counterexample method, the main points are as follows: [12]

The $\in$-image of the well-ordered structure $\langle A,<\rangle$ Assume that $<$ is a well ordering on $A$ and take for $\gamma(x, y)$ the formula: $y=r_{a n x}$ The transfinite recursion theorem then presents us with a unique function $E$ with domain $A$ such that for any $t \in A$ :

$$
E(t)=\operatorname{ran}(E\lceil\operatorname{seg} t)=E \llbracket \operatorname{seg} t \rrbracket=\{E(x) \mid x<t\}
$$

Let $\alpha=\operatorname{ran} E$; we will call $\alpha$ the $\in$-image of the well-ordered structure $\langle A,<\rangle$.

In the above example, $\alpha$ was a set equinumerous with $A$. Morever, while < was a well ordering on $A$, the membership relation $\epsilon_{\alpha}=\{x, y \in \alpha \times \alpha \mid x \in y\}$ provided a well ordering on $\alpha$.

Theorem: (a) let $<$ be a well ordering on $A$ and let $E$ and $\alpha$ be as described as above, $E(t) \notin E(t)$ for any $t \in A$

Proof [12] P. 182-183 we prove that $E(t) \notin E(t)$ by the least counterexample method. let $S$ be the set of counterexamples: $S=\{t \in A \mid E(t) \in E(t)\}$ We hope that $S$ is empty. But if not, then there is a least $\hat{t} \in S$. since $E(\hat{t}) \in E(\hat{t})$, there is some $s<\hat{t}$ with $E(\hat{t})=E(s)$. But then $E(s) \in E(s)$, contradicting the leastness of $\hat{t}$. hence $S=\varnothing$, which proves part (a).

The above discussion for a manifold is based on the morphological equation, wherein the $\llbracket J \rrbracket$ morphological element is used to describe the structure of the new phase $\alpha$, which corresponds to a manifold, but without discussing the structure of the parent phase, it is also an important structure in the material during phase transformation. The morphological equation is composed of two kinds of morphological elements $\llbracket J \rrbracket$ and $\llbracket H \rrbracket$ and a morphological transformation $|z|$, the $\llbracket H \rrbracket$ morphological element is used to describe the parent phase, and its structure and properties are different from the new phase produced from it. And in the phase transformation process it can produce a number of new phases, each of which is a manifold, they are developed by different embryos, but they are generally not connected, i.e., for any $i \neq k, \llbracket j_{i} \rrbracket \cap \llbracket j_{k} \rrbracket=\varnothing$, and their union sets can not be treated by union operation, need to apply disjoint union operation notation $\llbracket j_{i} \rrbracket \sqcup \llbracket j_{k} \rrbracket$, the whole set is disjoint union sets and with symbolic representation is $\sqcup_{i \in n} J(i)$, which can not be treated with 
continuous mapping or the homeomorphism method, because they're not in the same embryo of martensitic transformation.

Roughly speaking, a topological space is a geometric object, and the homeomorphism is a continuous stretching and bending of the object into a new shape. For example the martensitic transformation the new phase, a martensite plate $\alpha$ in an austenite grain $\gamma$ is a crystal, which may be also considered as a geo$\underline{\text { metric }}$ object in geometry or a manifold in algebraic geometry or topology, which shape can be treated by geometric or topological methods and can also be explained based on the structure $\llbracket J \rrbracket$. But there may exist many martensitic plate $\alpha$ in a same austenite grain $\gamma$, which have many different shape, size and orientation. The analysis of a geometric object, martensite plate $\alpha$, can be explained based on the structure $\llbracket J \rrbracket$, many different martensite plate $\alpha$, these new phases are belong to many different manifolds developed by different nucleus embryos, the whole union sets should be presented by disjoint union notation $\bigsqcup_{i \in n} J(i)$, which can not be treated with continuous mapping or the homeomorphism method. The structure of the phase transition is composed of a new phase and a parent phase, which can be expressed by the morphological equation, $M(n)=\llbracket H(n)|z(n)| \sqcup_{i \in n} J(i) \rrbracket$.

Hodge posed his conjecture: on a projective non-singular variety over $\mathrm{C}$, any class is a rational linear combination of classes $\mathrm{cl}(\mathrm{Z})$ of algebraic cycles. And he said that "I know of no example of a Kähler manifold whose fundamental 2-form $w$ is homologous to a multiple of an integral cycle, except the algebraic manifolds. There is surely a problem of great interest and importance here, which is a challenge to all interested in the theory of complex manifolds" [5].

The structure of parent phase, i.e., austenite grain $\gamma$ structure is extremely complex, it has not only many interior interface, and a exterior surface, the structure can be represented by a new formula, $\llbracket H(n)=M(n) \backslash|z(n)| \sqcup_{i \in n} J(i) \rrbracket$. So the parent phase structure $\llbracket H \rrbracket$ can not be treated with Hodge theory, there may be a Counterexample of the theory. Details will be discussed in my next articles.

\section{Concluding Remarks}

The image-math principle described by morphology equation can be used to explain the micro-image problem of material structure, which is the image-mathematical foundation of morphology physics.

There is a law of morphology in things or micro-images, that is, the law of the unity of $\llbracket H \rrbracket$ and $\llbracket J \rrbracket M E$ is the fundamental law of nature morphology, which is a law of qualitative structure of matter science and therefore also the fundamental law of thinking in images. This is the universality and absoluteness of the law of nature morphology. Each two kinds $\llbracket H \rrbracket$ and $\llbracket J \rrbracket$ of ME have their respective characteristics; in given conditions, two kinds of $\mathrm{ME}$ possess the common boundary $|z|$, and can coexist in a single entity and there exist complex action between two kinds of ME consequently can transform themselves 
into each other; this is the particularity and relativity of nature morphology.

A correct understanding of material structure must not only know which is made of $\llbracket J \rrbracket M E$ and the $\llbracket H \rrbracket M E$, but also how to construct the materials system from $\llbracket J \rrbracket$ and $\llbracket H \rrbracket$ ME. It represents a substantial advance in the understanding of correlated matter physics.

\section{Acknowledgements}

During the course of writing the paper, I had the benefit of many conversations with Academician Bang-Xin ZHOU, Prof. Gang WANG and Prof. Meiyi YAO, which often led to sudden clarification of an obscure point. To all of them, I wish to extend my sincere thanks.

\section{References}

[1] Bhadeshia, H.K.D.H. (2008) Mathematical Models in Materials Science. Materials Science and Technology, 24, 128-136. https://doi.org/10.1179/174328407X213107

[2] Xiao, J.M. (1995) Concise Discussion on Material. J. University of Science and Technology Beijing, 17, 303-314 (In Chinese).

[3] Qian, X.S. (1990) A New Discipline of Sciences-The Study of Open Complex Giant System and Its Methodology. Nature (In Chinese).

[4] van Der Waerden, B.L. (1945) Introduction to Algebraic Geometry. New York Dover pub.

[5] Hodge, W.V.D. (1950) The Topological Invariants of Algebraic Varieties. Proceedings ICM, AMS.

[6] Atiyah, M.F. and Hirzebruch, F. (1961) Analytic Cycles on Complex Manifolds. Topology, 1, X-45.

[7] Cattani, E., Deligne, P. and Kaplan, A. (1995) On the Locus of Hodge Classes. Journal of the American Mathematical Society, 8, No. 2. https://doi.org/10.1090/S0894-0347-1995-1273413-2

[8] Monastyrsky, M. (2001) Some Trends in Modern Mathematics and the Fields Medal.

[9] Zhou, Z.Q. (2006) Mathematical Model for Morphological Element of Higher-Order Ferrite. Materials Science and Engineering A, 438-440, 166-169. https://doi.org/10.1016/j.msea.2006.02.195

[10] Zhou, Z.Q. (2011) Reconstruction and Decision of 3D Model of Higher-Order Microstructures. Proceedings of 2011 World Congress on Engineering and Technology, 4, 739-742.

[11] Munkres, J.R. (2000) Topology. Pearson Education, 392.

[12] Enderton, H.B. (2006) Elements of Set Theory. Elsevier Science, 222.

[13] Hamilton, A.G. (2003) Logic for Mathematicians. Cambridge University Press in 1988.

[14] Clapham, C. (1996) Oxford Concise Dictionary of Mathematics.

[15] Zhou Yi (Book of Change) (2000) Ma Hengchang Comments. Huaxia Publishing Press (In Chinese).

[16] Deligne, P. The Hodge Conjecture. Clay Mathematics Institute.

[17] Grothendieck, A. (1968) The Hodge General Conjecture Is False for Trivial Reasons. Topology, 8, 299-363. https://doi.org/10.1016/0040-9383(69)90016-0 